Further delineation of an entity caused by CREBBP and EP300 mutations but not resembling

\title{
Rubinstein-Taybi syndrome
}

Leonie A. Menke, ${ }^{1}$ the DDD study, ${ }^{2}$ Thatjana Gardeitchik, ${ }^{3}$ Peter Hammond, ${ }^{4}$ Ketil Riddervold Heimdal, ${ }^{5}$ Gunnar Houge, ${ }^{6}$ Sophia B. Hufnagel, ${ }^{7}$ Jianling Ji, ${ }^{8}$ Stefan Johansson, ${ }^{6}$ Sarina G. Kant, ${ }^{9}$ Esther Kinning, ${ }^{10}$ Eyby Leon, ${ }^{8}$ Ruth Newbury-Ecob, ${ }^{11}$ Stefano Paolacci, ${ }^{12}$ Rolph Pfundt, ${ }^{3}$ Nicola K Ragge, ${ }^{13}$ Tuula Rinne, ${ }^{3}$ Claudia Ruivenkamp, ${ }^{9}$ Sulagna C Saitta, ${ }^{8}$ Yu Sun, ${ }^{14}$ Marco Tartaglia, ${ }^{12}$ Paulien Terhal, ${ }^{15}$ Anthony J. van Essen, ${ }^{16,+}$ Magnus Dehli Vigeland, ${ }^{5}$ Bing Xiao, ${ }^{14}$ Raoul C. Hennekam $^{1}$

${ }^{1}$ Department of Pediatrics, Academic Medical Center, Amsterdam, The Netherlands

${ }^{2}$ Wellcome Trust Sanger Institute, Wellcome Genome, Campus, Hinxton, Cambridge, United Kingdom

${ }^{3}$ Department of Human Genetics, Radboud University Medical Center, Nijmegen, The Netherlands

${ }^{4}$ Big Data Institute and Nuffield Department of Obstetrics and Gynaecology, University of Oxford, Oxford, United Kingdom

${ }^{5}$ Department of Medical genetics, Oslo University Hospital, Oslo, Norway

${ }^{6}$ Center for medical genetics and molecular medicine, Haukeland University Hospital, Bergen, Norway

${ }^{7}$ Division of Genetics and Metabolism, Children's National Health System, Washington, USA 
${ }^{8}$ Division of Genomic Medicine, Department of Pathology, Childrens Hospital Los Angeles and Keck USC School of Medicine

${ }^{9}$ Department of Clinical Genetics, Leiden University Medical Center, Leiden, The Netherlands

${ }^{10}$ West of Scotland Genetics Service, Queen Elizabeth University Hospitals, Glasgow

${ }^{11}$ Department of Clinical Genetics, University Hospitals Bristol, Bristol

${ }^{12}$ Genetics and Rare Diseases Research Division, Ospedale Pediatrico Bambino Gesù, Rome, Italy

${ }^{13}$ Birmingham Women's Hospital, Birmingham, United Kingdom

${ }^{14}$ Department of Pediatric Endocrinology/Genetics, Xinhua Hospital, Shanghai Jiao Tong University School of Medicine, Shanghai Institute for Pediatric Research, Shanghai, China

${ }^{15}$ Department of Genetics, Wilhelmina Children's Hospital, Utrecht, The Netherlands

${ }^{16}$ University of Groningen, University Medical Center Groningen, Department of Genetics, Groningen, The Netherlands

${ }^{\dagger}$ Deceased

Running title: CREBBP and EP300 mutations causing non-RSTS phenotype

\section{Corresponding author}

Dr LA Menke, Department of Pediatrics, H7-217, Academic Medical Center, Meibergdreef 9, 1105 AZ Amsterdam, The Netherlands, Email: I.a.menke@amc.nl, Tel + 31 (0)205668174, Fax + $31(0) 205669683$ 
Menke et al.

\section{Key words}

CREBBP; CBP; P300; EP300; exon 30; exon 31; exome sequencing; intellectual disability, Rubinstein-Taybi syndrome; RSTS; syndrome; mutation; clinical features; case series; genotypephenotype correlation 


\begin{abstract}
We have described in 2016 that missense variants in parts of exons 30 and 31 of CREBBP can cause a phenotype that differs from Rubinstein-Taybi syndrome (RSTS). Here we report on another thirteen patients, eleven with a variant in this specific region of $C R E B B P$ (between base pairs 5128 and 5614) and two with a variant in the homologous region of EP300. None of the patients show the characteristics typical for RSTS. The variants have been detected by exome sequencing using a panel for intellectual disability in all but one individual, in whom the entity was recognized clinically and Sanger sequencing was performed. The main characteristics of the patients are an apparent developmental delay (90\%), autistic behavior (65\%), short stature (42\%) and microcephaly (43\%). Medical problems consist predominantly of feeding problems (71\%), vision (50\%) and hearing (54\%) impairments, recurrent upper airway infections (38) and epilepsy (24\%). Major malformations are uncommon and include cryptorchidism (50\% of males), congenital heart anomalies (17\%), malformations of the intestines (13\%) and cleft palate (8\%). Brain malformations were limited to a small or absent callosal body. First analysis of the facial characteristics indicates that individuals with a variant between base pairs 5595 and 5614 of CREBBP show a specific phenotype (ptosis, telecanthi, ptosis, short palpebral fissures, depressed nasal ridge, short nose, anteverted nares, short columella, and long philtrum). Normative inversion of 3D face shape demonstrated resemblance to individuals with a duplication of $16 \mathrm{p} 13.3$ (the region that includes CREBBP), possibly indicating a gain of function. The other affected individuals show a less specific phenotype. We conclude that there is now more firm evidence that variants in these specific regions of $C R E B B P$ and $E P 300$ result in a phenotype that differs from RSTS, and that this phenotype may be heterogeneous.
\end{abstract}




\section{INTRODUCTION}

In 2016, we reported on eleven patients with intellectual disability in whom a missense mutation was detected in a specific CREBBP region [Menke et al., 2016]. Although CREBBP variants are well-known to cause of Rubinstein-Taybi syndrome (RSTS) [Petrij et al., 1995], none of the patients had the typical characteristics of RSTS. Several but not all patients had short palpebral fissures, telecanthi, a depressed nasal ridge, short nose, anteverted nares, short columella and long philtrum [Menke et al., 2016]. Other symptoms were autistic behavior, recurrent upper airway infections, feeding problems and impaired hearing.

Another eleven patients with a mutation in the same region of CREBBP have now been detected. The molecular studies consisted of exome sequencing and Sanger sequencing in one patient, who was clinically recognized as showing marked resemblance to the earlier reported patients. Additionally, we detected a variant in the homologous region of EP300 in one patient and gathered additional information of one other previously reported patient with an EP300 variant in the same region [Hamilton et al., 2016]. None of the patients showed the characteristics typical for RSTS. Here, we report on the phenotypes and genotypes of the above patients, in order to further delineate the phenotype. Additionally, we performed normative inversion of a three-dimensional (3D) image [Hammond et al., 2014] to evaluate the overlapping facial features of several patients with those of patients with a duplication of 16p13.3. 


\section{MATERIALS AND METHODS}

All patients had been referred for clinical genetic evaluation because of (apparent) intellectual disability and/or behavioral problems. Exome sequencing was performed in all patients in whom no clinical diagnosis was suggested. In one individual (patient C19), the resemblance to the earlier reported phenotype [Menke et al., 2016] was clinically recognized, and directed Sanger sequencing of CREBBP was performed. Exome sequencing was performed either by using a panel targeted for genes known to be mutated in patients with intellectual disability, or by untargeted trio analyses comparing results of exome sequencing in the affected child to those of his/her parents. In the latter, both Mendelian conditions with an autosomal dominant, autosomal recessive, or X-linked pattern of inheritance were evaluated. The mutations were confirmed by Sanger sequencing, except for patients C12 and E2 in whom the high coverage of the exome study yielded a sufficiently reliable result to use the exome result for patient care purposes. Paternity was confirmed in all patients (Nicky, Ruth, Esther, Rolph and Tuula; could you please verify whether this is true for your patients?), except for patient C16 whose parents had deceased prior to evaluation, and in patient C19. The effect of the missense variants was predicted using three different in silico prediction programs: Polyphen2 [Adzhubei et al., 2010], SIFT2 [Ng et al., 2003], and MutationTaster [Schwarz et al., 2010]. We also determined the presence of the variants in two population cohorts (Exome Sequencing Project (ESP) and Exome Aggregation Consortium (gnomAD) . The Human Gene Mutation Database (HGMD) [Stenson et al., 2014], the Leiden Open Variation Database (LOVD) [Fokkema et al., 2011], and personal registries of one of us (RCH) were searched for patients with the clinical diagnosis of RSTS and a 
missense mutation in the regions of $C R E B B P$ and $E P 300$ in which the patients presented here have mutations.

We gathered clinical and molecular data, and two authors (LAM; RCH) scored the facial and distal limb morphology of all patients as provided by the clinician, to achieve uniform scoring. Written informed consent for publication of clinical features and/or photographs were obtained from parents for all patients.

Using the Vectra M3 system and a facial 3D-camera (both provided by Canfield Scientific), a 3D photogrammetric image was captured of patient $\mathrm{C} 11$, one of the previously published patients [Menke et al., 2016], and compared with that of a previously published Caucasian patient with a duplication of 16p13.3 [Hammond et al., 2014]. Subsequently, the face shape of patient C11 was inverted relative to a matched norm to produce an opposite form as described elsewhere [Hammond et al., 2014]. In short, we used dense surface modelling techniques to match the face to a facial norm of unaffected individuals of matched age, sex and ethnicity, and then reversed the individual's face shape differences from the matched norm to produce the normative inversion. The inverted face was subsequently compared with the mean face of 13 Caucasian patients with RSTS of North-Western European ancestry with a proven mutation or deletion of CREBBP.

The Medical Ethics Committee of the AMC in Amsterdam has granted permission to perform the study. 


\section{RESULTS}

\section{Phenotype}

We compare the main morphological data of the present 13 patients to the 11 earlier reported patients in Table I, and their medical problems are compared in Table II. The phenotypes are illustrated in Figures 1 and 2, and the 3D image analysis is presented in Figure 3. Below, we provide a short description of each patient, providing only data that are not available in the tables.

Patient C12 vomited easily in the first 3 years of life, and had such swallowing problems that he could only take fluids or puréed food. Recurrent upper airway infections made adenoidectomy necessary. As a toddler, he suffered from severe constipation. He was quite thin in his first years, but developed obesity later on. At 10 years, he was tested using SON-R 5.5-17 and found to have an IQ of 50. His parents described him as a friendly and content boy, who liked routines and being on his own. He could easily get upset if unable to verbalize his thoughts.

Patient C13 was originally thought to have symptoms resulting from a monosomy 20 mosaicism (1.5-6.5\% monosomy 20 in blood cells) which was reported as such [Hochstenbach et al., 2014]. He had feeding difficulties and swallowing problems resulting in a relatively low weight until the age of 8 , after which he progressively developed obesity, and striae. At 10 years he scored a total IQ of 54 on the WISC-III (verbal IQ 50, performal IQ 62). From 13 years on he was treated for hypothyroidism. He had decreased mobility of the palms, hammertoes 
and pes cavus of predominantly the right foot, and had unexplained muscle weakness and fatigue after exercise, using a wheelchair for distances taking longer than a 30 minute walk [Hochstenbach et al., 2014]. The Achilles tendons of his feet had been surgically elongated. His behavior was friendly and social. He had an inability to express sadness. As his development did not fit in with other individuals with monosomy 20 , exome sequencing was performed to check for a further explanation of his development.

Patient C14 had severe failure to thrive and feeding difficulties being unable to chew and needing puréed food in the first years of life. In her teens, she had surgery for a tethered spinal cord. Next to the findings mentioned in the table, she also had a supernumerary nipple. At 16 years, she was a cheerful girl, nonverbal, displaying unusual hand movements, and who liked routines. At 19 years she had sensory processing difficulties and scored a developmental age of 5 (expressive language) to 15 months (gross motor skills) using the Bayley Scales of Infant and Toddler Development-Third Edition.

Patient C15 had severe failure to thrive. She did not pass the hearing test, but responded to sounds. She had repetitive, stereotypic hand movements, such as wringing.

Patient C16 had significant aggressive and self-injurious behavior problems from infancy onwards and was institutionalized at the age of 6 years. His main problem had always been his behavior, which received several different diagnoses but was recently recognized as Multiple Complex Developmental Disorder, a subtype of autism with generalized anxieties. Over the years, several psychotropic medications had been prescribed with varying degrees of effect. The combined use of routines, a calm and comforting approach, as well as sodium valproate and citalopram rendered the best results. A WISC-R test at the age of 37 showed an intelligence 
equivalent with a developmental age of 7 years. At the age of 43 , the Vineland adaptive behavior scale showed developmental ages being 4, 5 and 9 years for communication (receptive, expressive and written, respectively), 6 years for daily living skills and 3 5/12 for socialization. An orchiectomy was performed at the age of 33. At 44 years, he had a gastric perforation and ileus, from which he recovered completely. He developed gynecomastia and osteopenia due to low androgen levels for which denosumab was started. In his fifties he gradually developed a cataract, which was surgically corrected at the age of 56 . Next to the CREBBP variant he also had a duplication of $9 \mathrm{q} 34.3$, which included CACNA1B and part of EHMT1. It remains uncertain whether or not this chromosome imbalance has an influence on the phenotype.

Patient C17 had preaxial polydactyly of the left hand which was surgically corrected. He did not yet walk independently nor used words at 2 years of age. He did not pass the hearing test, but responded to sounds. Ophthalmic examination showed exudative vitreoretinopathy in the right eye which segregated as separate trait in the family. Two years after a surgical procedure, his eye responded to light. No behavioral problems were noted.

Patient C18 had an increased nuchal translucency noticed during pregnancy. At birth, he was found to have a large cleft palate. He had a limited ability to open his mouth (Esther, is it known what the cause was?) for which surgery was performed. He showed autistic traits including limited eye contact, liking of routine, and obsessions, and is awaiting formal psychological assessment. He had ... (what were the complaints that made the physicians search for CF?) urging for further studies which allowed the (molecularly confirmed) diagnosis of cystic fibrosis. 
Patient C19 had an abnormally soft/raspy cry at birth. She had failure to thrive, multiple food allergies, and an intestinal malrotation that required surgical correction. Several maternal family members were known to have had malrotation in infancy. The girl was noted to have pseudo-papiledema, without visual complaints. Her facial features reminded the physician of another patient with a CREBBP missense mutation (patient 10 in Menke et al., 2016), which suggested Sanger sequencing of CREBBP for the molecular confirmation. (Sophia, would you please let us know whether you have seen the patient again last autumn - I read in your letter that you were planning to do so - and if yes, would you please add extra information to the above as well as in table II?)

Patient C20 had a fine motor delay and a sensitivity for food texture. He displayed difficulties with social communication and emotional dysregulation with temper tantrums. He had learning delays with memory difficulties and attention problems. He was found to have an autism spectrum disorder and a full-scale IQ of 108 (WISC-III). An MRI of the brain showed overall thinning in the paracentral and superior parietal lobule and generalized white matter loss. Apart from the mutation in $C R E B B P$, a maternally inherited variant of unknown significance was found in COL5A2. However, his mother did not show obvious symptoms of Ehlers-Danlos syndrome, and it is unclear whether his pectus carinatum and joint hypermobility can be attributed to this variant.

Patient C21 had multiple malformations (Table II) as well as congenital contractures of both large and small joints which gradually improved over time. She had a severe developmental delay. She developed a tendency for self-injurious behavior. From the age of 4 
years, she suffered increasingly from epilepsy and central apneas. She died at the age of 5 as a result of an apneic incident.

In patient C22, a ventricular septum defect and pulmonic stenosis were detected prenatally. Because of progressive heart failure the defects were surgically corrected at 3 days of age. At 6 months of age, he had a subileus due to a stenotic segment of the colon that was resected. He was able to use a few words and was described as hyperactive and generally a happy and content child.

Patient $\mathrm{E} 1$ has been reported before in a series of patients with EP300 variants [Hamilton et al., 2016]. As a neonate, she was floppy with very lax joints. Feeding problems, including difficult swallowing, necessitated a percutaneous gastrostomy (PEG). She had recurrent otitis, airway infections and urinary tract infections, and was found to have low immunoglobulins. She had bilateral moderate sensorineural hearing loss. In her teens, she had ulcers in the duodenum thought possibly to fit Crohn disease but biopsies were inconclusive. She was diagnosed with autism spectrum disorder, showed hyperactive behaviors and suffered insomnia.

Patient E2 slept unusually much during infancy, did not seem to notice feelings of hunger and had no sucking reflex. At the age of 13 , she still did not seem to notice feeling of hunger and preferred pureed food. At 4 years, speech therapy was started both for speech delay and for swallowing problems, and at 5 years, physiotherapy was started because of a delay in fine motor skills. She was psychometrically tested and found to have an IQ of 91 (verbal 101, performal 82). Special education was initiated at 9 years of age due to a slow work pace and poor memory skills. Furthermore, autism spectrum disorder was diagnosed. Presently she 
continues to have recurrent otitis, a decrease in muscle strength of the hands, and has developed a progressive contracture of the $5^{\text {th }}$ fingers in the last two years.

\section{Genotype}

The genotypes of the present patients are tabulated in Table III and compared to earlier reported patients with Rubinstein-Taybi syndrome and a CREBBP or a EP300 mutation in Fig. 4. All mutations are de novo. All CREBBP variant are clustered at the end of exon $30(n=1)$ and the first part of exon 31 ( $n=10$ ), between base pairs 5,155 and 5,614 (codons 1,719 to 1,872 ). The 2 variants in EP300 were located in the homologous areas, between base pairs 5,582 and 5,602 (codons 1,861 and 1,868; Fig. 4a-b). The conservation of these areas among orthologues in CREBBP and EP300 are as depicted elsewhere (https://www.ncbi.nlm.nih.gov/homologene). Some variants were located in or near a zinc finger domain, but most variants were located in a domain with unknown function. All variants changed a highly conserved amino acid, and all were predicted to be pathogenic by three different in silico prediction programs. None of the variants had been reported before (HGMD/LOVD; ESP; gnomAD).

\section{D-scanning}

When comparing the 3D-dimensional image of patient C11, one of the previously published patients [Menke et al., 2016], with that of a previously published Caucasian patient with a duplication of 16p13.3 [Hammond et al., 2014], a number of similarities were found. Both have a long face, upslanted palpebral fissures, inverted nares, a short columella and long philtrum. Likewise, the inverted face of patient C11 shared similarities with the 'mean RSTS face' 
consisting of a broad face, downslanting palpebral fissures, low hanging columella, short philtrum and micro/retrognathia.

\section{DISCUSSION}

The present study adds to the earlier reported series of eleven patients with a mutation in the last part of exon 30 or the beginning of exon 31 of $C R E B B P$, and two patients with a mutation in the homologous region of EP300. CREBBP and EP300 mutations are well-known causes of Rubinstein-Taybi syndrome (RSTS), but none of the patients had typical characteristics of RSTS. The present study confirms a previous one [Menke et al., 2016]: patients with missense mutations in this region of CREBBP show a phenotype that differs substantially from that in RSTS patients with mutations elsewhere in CREBBP. The finding that also patients with mutations in the homologous region of EP300 do not show an RSTS phenotype, underscores this conclusion.

The present study also confirms that the phenotype of the patients is heterogeneous, and only patients with a mutation at the $3^{\prime}$ end of CREBBP between base pairs 5595 and 5614 (patients C9, C10, C11, C15, C17, C18, C19, C21, and C22) share facial similarities (Fig. 1). Patient $\mathrm{C} 9, \mathrm{C} 10, \mathrm{C} 17, \mathrm{C} 18$ and $\mathrm{C} 19$ share the same mutation, and patient $\mathrm{C} 11$ and $\mathrm{C} 22$ also have the same mutation. Facial characteristics are ptosis, telecanthi, ptosis, short palpebral fissures, depressed nasal ridge, short nose, anteverted nares, short columella, and long philtrum. The facial characteristics change with age and the phenotype is best recognized during infancy (Fig. 1c). The phenotype in patients in the $5^{\prime}$ part of the region is less specific, and numbers are too small to draw any conclusions at the present. This is also the case for the EP300 mutated 
patients (patients E1 and E2). The phenotype in patient C16 differs which may be caused by the concomitant duplication of chromosome region 9q34.3.

None of the previously reported patients [Menke et al., 2016] and the presently reported patients have the facial characteristics of RSTS, including the universally present grimacing smile, and none truly broad and/or angulated thumbs or halluces. The patients share other characteristics, including intellectual disability of variable severity (which constitutes a bias as it had been the reason for exome sequencing), and in most short stature and microcephaly (Table II). In several patients, autism or a behavior resembling autism is present, and several patients have visual and/or hearing impairments. Other symptoms are feeding problems, epilepsy, recurrent upper airway infections, and scoliosis and/or kyphosis. There are only few patients with malformations of which the most important ones are cleft palate, congenital heart anomaly, renal anomaly, malrotation, and cryptorchidism. Hip dysplasia, hypermobility and contractures have been infrequently found. Sandal gaps, unusual deviation of the distal phalanx of the halluces and partial cutaneous syndactyly are other findings, and several patients have long, slender fingers. A single patient has preaxial polydactyly. Findings at brain MRI are few: absent corpus callosum, enlarged ventricles and cerebral atrophy.

The behavioral characteristics of patients with variants in this region of CREBBP / EP300 are at present not sufficiently studied. We will use the recently built web-based database 'Waihonapedia' [Baas et al., 2015] for this. This system consists of an initial extensive questionnaire for background cross-sectional data, and subsequent follow-up using small questionnaires, with a particular focus on behavioral aspects [de Winter et al., 2016]. 
The borders of the site CREBBP/EP300 variants causing an RSTS phenotype and the variants in CREBBP/EP300 in the earlier and here reported patients are located between base pair 5094 (unpublished patient with RSTS) and base pair 5128 at the 5'end; and between base pair 5614 and 5641 (unpublished patient with RSTS) at the 3' end of CREBBP. Reports of additional patients may further refine these borders. We expect that the borders for EP300 mutations will be situated at the sites homologous to CREBBP, but the number of known patients is at present too small to determine this.

CREBBP and EP300 are central nodes in eukaryotic transcriptional regulatory networks [Dyson et al., 2016]. They interact with more than 400 transcription factors and other regulatory proteins [Ramos et al., 2010]. They were suggested to be the "molecular interpreters that can parse and/or conjugate the regulatory words, phrases, and sentences of the genome" [Smith et al., 2004]. Part of this ability stems from the presence of long, intrinsically disordered regions between the various CREBBP/EP300 interaction domains [Dyson et al., 2016]. The mutation cluster at the 3 'end of the present and previous report falls outside a functional domain and are thus located in these regions. The facial features of the presently reported patients resemble those of patients with a duplication of CREBBP (Fig. 3). Likewise, the inverted image produced using a 3D scan resemble the facial characteristics of RSTS. Our hypothesis is that missense variants in the last part of exon 30 and beginning of exon 31 of CREBBP/EP300 result in a gain of function. In contrast, variants in other parts of $C R E B B P / E P 300$, causing RSTS, result in haplo-insufficiency or perturb the function of a specific domain, typically the HAT domain. As not all functions of CREBBP/EP300 are dose-dependent, an inversed phenotype is 
not expected for each sign of the two phenotypes. Functional studies are in preparation to study the consequences of the presently reported missense mutations in detail.

The present cluster of variants overlaps with the ZNF2 (Zinc finger, ZZ-type; residues 1,701 to 1,742) and ZNF3 (Zinc finger, TAZ-type; residues 1,766 to 1,844) domains. Both domains contain cysteine residues that mediate $\mathrm{Zn}^{2+}$ binding to stabilize helical folding and mediate interactions with numerous transcriptional regulatory proteins [De Guzman et al., 2000; Ponting et al., 1996]. These data suggest that this cluster of variants affects the binding properties of the two zinc finger domains to CREBBP partners by affecting their proper folding. Additional functional studies are needed to prove this hypothesis.

The previous [Menke et al., 2016] and present report describe in total 24 individuals with this phenotype and a missense mutation in a relatively limited part of $C R E B B P / E P 300$. This group of individuals were referred to, or seen by, us in a relatively short period of time ( 2.5 years). Furthermore, all but one of the patients were recognized by using exome sequencing with a panel targeted to detect variants in genes known to cause intellectual disability, an approach that is used frequently in only a limited number of countries. Therefore, it seems this entity is not extremely rare. The present report also confirms that the combined experience of individual clinicians and molecular geneticists is essential to recognize that a specific group of variants is likely causal for the phenotype, and to define the characteristics of such entities. The importance of databases containing detailed information of both phenotype and genotype, and the need for international collaborations cannot be overstated.

We conclude that the present study confirms that individuals with missense mutations in the last part of exon 30 and the beginning of exon 31 of CREBBP show a phenotype that 
differs substantially from that in RSTS patients who have mutations elsewhere in CREBBP. It also shows that similarly such mutations in the homologous region of EP300 cause a phenotype differing from RSTS. The phenotype shows heterogeneity and likely constitutes at least two different entities. We plan to delineate the phenotypes in more detail by including more patients, perform detailed behavioral studies, and initiate functional studies to determine the pathogenesis. 


\section{ACKNOWLEDGEMENTS}

We are pleased to thank all families for their generous participation. The DDD study presents independent research commissioned by the Health Innovation Challenge Fund (grant number HICF-1009-003) see full acknowledgement as previously reported [Deciphering Developmental Disorders, 2015]. MT obtained funding from the Ministry of Health (RC2016) and Bulgari (GeneRare). LM obtained funding from the Rare Disease Foundation and the BC Children's Hospital Foundation.

\section{INTERNET RESOURCES}

SMART, http://smart.embl-heidelberg.de/; HOMOLOGENE, https://www.ncbi.nlm.nih.gov/homologene

http://www.ebi.ac.uk/interpro/protein/Q92793 


\section{REFERENCES}

Adzhubei IA, Schmidt S, Peshkin L, Ramensky VE, Gerasimova A, Bork P, Kondrashov AS, Sunyaev SR. 2010. A method and server for predicting damaging missense mutations. Nat Methods 7:248-249. Baas M, Huisman S, van Heukelingen J, Koekkoek G, Laan HW, Hennekam RC. 2015. Building treasures for rare disorders. Eur J Med Genet 58:11-13.

De Guzman RN, Liu HY, Martinez-Yamout M, Dyson HJ, Wright PE. 2000. Solution structure of the TAZ2 (CH3) domain of the transcriptional adaptor protein CBP. J Mol Biol 303:243-253.

de Winter CF, Baas M, Bijlsma EK, van Heukelingen J, Routledge S, Hennekam RC. 2016. Phenotype and natural history in 101 individuals with Pitt-Hopkins syndrome through an internet questionnaire system. Orphanet J Rare Dis 11:37.

Deciphering Developmental Disorders S. 2015. Large-scale discovery of novel genetic causes of developmental disorders. Nature 519:223-228.

Dyson HJ, Wright PE. 2016. Role of Intrinsic Protein Disorder in the Function and Interactions of the Transcriptional Coactivators CREB-binding Protein (CBP) and p300. J Biol Chem 291:6714-6722.

Fokkema IF, Taschner PE, Schaafsma GC, Celli J, Laros JF, den Dunnen JT. 2011. LOVD v.2.0: the next generation in gene variant databases. Hum Mutat 32:557-563.

Hamilton MJ, Newbury-Ecob R, Holder-Espinasse M, Yau S, Lillis S, Hurst JA, Clement E, Reardon W, Joss S, Hobson E, Blyth M, Al-Shehhi M, Lynch SA, Suri M, Study DDD. 2016. Rubinstein-Taybi syndrome type 2: report of nine new cases that extend the phenotypic and genotypic spectrum. Clin Dysmorphol 25:135-145.

Hammond P, McKee S, Suttie M, Allanson J, Cobben JM, Maas SM, Quarrell O, Smith AC, Lewis S, Tassabehji M, Sisodiya S, Mattina T, Hennekam R. 2014. Opposite effects on facial morphology due to gene dosage sensitivity. Hum Genet 133:1117-1125.

Hochstenbach R, Krijtenburg PJ, van der Veken LT, van der Smagt J, Roeleveld-Versteegh A, Visser G, Terhal P. 2014. Monosomy 20 mosaicism revealed by extensive karyotyping in blood and skin cells: case report and review of the literature. Cytogenet Genome Res 144:155-162.

Menke LA, van Belzen MJ, Alders M, Cristofoli F, Study DDD, Ehmke N, Fergelot P, Foster A, Gerkes EH, Hoffer MJ, Horn D, Kant SG, Lacombe D, Leon E, Maas SM, Melis D, Muto V, Park SM, Peeters H, Peters DJ, Pfundt R, van Ravenswaaij-Arts CM, Tartaglia M, Hennekam RC. 2016. CREBBP mutations in individuals without Rubinstein-Taybi syndrome phenotype. Am J Med Genet A 170:2681-2693. Ng PC, Henikoff S. 2003. SIFT: Predicting amino acid changes that affect protein function. Nucleic Acids Res 31:3812-3814.

Petrij F, Giles RH, Dauwerse HG, Saris JJ, Hennekam RC, Masuno M, Tommerup N, van Ommen GJ, Goodman RH, Peters DJ, et al. 1995. Rubinstein-Taybi syndrome caused by mutations in the transcriptional co-activator CBP. Nature 376:348-351.

Ponting CP, Blake DJ, Davies KE, Kendrick-Jones J, Winder SJ. 1996. ZZ and TAZ: new putative zinc fingers in dystrophin and other proteins. Trends Biochem Sci 21:11-13.

Ramos YF, Hestand MS, Verlaan M, Krabbendam E, Ariyurek Y, van Galen M, van Dam H, van Ommen GJ, den Dunnen JT, Zantema A, t Hoen PA. 2010. Genome-wide assessment of differential roles for p300 and CBP in transcription regulation. Nucleic Acids Res 38:5396-5408.

Schwarz JM, Rodelsperger C, Schuelke M, Seelow D. 2010. MutationTaster evaluates disease-causing potential of sequence alterations. Nat Methods 7:575-576.

Smith JL, Freebern WJ, Collins I, De Siervi A, Montano I, Haggerty CM, McNutt MC, Butscher WG, Dzekunova I, Petersen DW, Kawasaki E, Merchant JL, Gardner K. 2004. Kinetic profiles of p300 occupancy in vivo predict common features of promoter structure and coactivator recruitment. Proc Natl Acad Sci U S A 101:11554-11559.

Stenson PD, Mort M, Ball EV, Shaw K, Phillips A, Cooper DN. 2014. The Human Gene Mutation Database: building a comprehensive mutation repository for clinical and molecular genetics, diagnostic testing and personalized genomic medicine. Hum Genet 133:1-9. 


\section{FIGURE LEGENDS}

Fig. 1. Facial morphology of the presently described patients with a CREBBP or EP300 variant in exon 30/31. Frontal $(A)$ and lateral (B) views, and evolution of the facial characteristics with age in 8 patients (C). Patient numbers correspond to those in text, tables and other figures. Detailed description of facial and distal limb morphology can be found in Table I. Patients show similarities and differences. Especially patients with mutations distal to codon 5,595 (patients C15 and above) resembled one another markedly. Characteristics are telecanthi, short and upslanted palpebral fissures, depressed nasal ridge, short nose, anteverted nares, short columella and long philtrum. The faces of patients C14 and below are less specific. The ears of patients C15, C17, C19 and C21 were low-set and had an overfolded helix. The facial changes with ages demonstrate that characteristics are best recognized during infancy. The depressed nasal bridge and ridge become less marked with age.

Fig. 2. Distal limb morphology of the presently described patients with a CREBBP or EP300 missense variant in exon $30 / 31$.

Patient numbers correspond to text, tables and other figures. Detailed description of the signs can be found in Table I. A. Hands. Patients C14, E1, C16 and E2 had long, slender fingers. Patient E2 had a fixed flexion of the pinks. B. Feet. Note that none of the patients had broad or angulated thumbs, nor broad distal phalanges of the fingers, as seen in patients with Rubinstein-Taybi syndrome. The halluces of patients C17 and C19 were broad, but not angulated. Patient E1, C17 and C19 showed fibular deviation of the distal phalanx of the hallux; patients E1, C16, C19 and C20 had sandal gaps; and patient E1, C15, C17 and C21 
had partial cutaneous syndactyly. Patient C13 had pes cavus, predominantly of the right foot, with hammertoes and underdeveloped calve muscles.

Fig. 3. Distribution of CREBBP and EP300 Domains and Mutations in Present and Previous Patient Cohort (in red, on top) versus Mutations in RSTS Patients (in blue, beneath). Schematic representation of the $C R E B B P(A)$ and $E P 300$ (B) protein and its functional domains. Deducted from http://www.ebi.ac.uk/interpro/protein/Q92793. Mutations from http://chromium.lovd.nl/lovd2/home.php?select $\mathrm{db}=C R E B B P$ and personal registries of one of the authors $(\mathrm{RCH})$. Previous patient cohort refers to the cohort published previously [Menke et al., 2016].

Fig. 4. 3-Dimensional images of a patient with the new entity, normative inversion of her face and comparison with dup 16p13.3 and RSTS patients with an CREBBP mutation. 20170214 depicts the 3D face shape of an earlier described patient (patient C11) with a mutation in exon 31 of CREBBP [Menke et al., 2016]; INV_20170214 shows the normative inversion of her face shape; dup 16p13.3 shows a Caucasian patient with a duplication of the region $16 \mathrm{p} 13.3$ (including CREBBP), and RTS shows the average face surface constructed using 13 Caucasian molecularly confirmed RSTS patients. The signature heat map highlights in red-green-blue spectrum the regional differences (compression-coincidence-expansion) from the average of faces of 50 healthy controls. Note the similarities between the face of 20170214 and the dup 16p13.3 patient in their long face, upslanted palpebral fissures, inverted nares, short columella and long philtrum. Likewise, note the similarities of her inverted face of 20170214 with the 'mean RSTS face' consisting of a broad face, 
Menke et al.

downslanting palpebral fissures, low hanging columella, short philtrum and micro/retrognathia. 